\title{
Stabilization for Markovian Jump Systems with Additive Time-varying Delays
}

\author{
Haiyang Zhang ${ }^{1}$, Zhipeng Qiu ${ }^{1}$, Lianglin Xiong ${ }^{2, *}$ and Yanmeng Wang ${ }^{3}$ \\ ${ }^{1}$ School of Science, Nanjing University of Science and Technology, Nanjing 210094, China \\ ${ }^{2}$ School of Mathematics and Computer Science, Yunnan Minzu University, Kunming 650500, China \\ ${ }^{3}$ College of Sciences, Nanjing University of Aeronautics and Astronautics, Nanjing 210016, China \\ ${ }^{*}$ Corresponding author
}

\begin{abstract}
In this paper, the problem of stabilization for Markovian jump systems with additive time-varying delays is considered. First of all, by constructing a new Lyapunov functional with triple integral terms, using new inequalities, convex combination technique and combining with other analytical techniques, the stochastically stability and stabilization conditions of the Markovian jump systems in terms of linear matrix inequalities with lower conservatism are obtained. Then, the controller of the closed-loop system is designed by the transformation technique of inequalities. Finally, a numerical example is given to verify the effectiveness of the provided method and the superiority of the results.
\end{abstract}

Keywords-markovian jump systems; additive time-varying delays; stochastically stabilization; linear matrix inequalities

\section{INTRODUCTION}

In recent years, a large number of attentions have been focused on the study of the Markov jump systems (MJSs), since this family of systems were firstly introduced by Krasovskii and Lidskii[1]in 1961. Because of their advantage of modeling many practical dynamic systems, lots of works on stability analysis, controller synthesis and filter design have been focused on the study for MJSs [2-8]. Meanwhile, as a special kind of hybrid systems with finite operation modes whose structures are subject to random abrupt changes, which may result from abrupt phenomena such as random failures and repairs of the components, sudden environmental changes, changes in interconnections of subsystems, etc. So, the research about the synthesis problem for MJSs will become more complex and difficult. In addition, delay systems are frequently encountered in various practical systems, such as engineering, biology, economics, neural networks, and network control, [916], however, this class system is instability and performance deterioration in many cases. Thus, recent past decades have witnessed extensive research on delay systems in the literature, including stability analysis, stabilization $\mathrm{H} \infty$ controller design, robust filtering analysis, and model reduction or simplification [17-26].

Especially, the delay systems with additive time-varying delay component were firstly proposed in [27], which has a strong application background in remote control and networked control (see, for instance[18,28,29] and the references therein). Take a state-feedback networked control, for example. Some time delays will appear inevitably when signals are transmitted from one device to another, due to the physical plant, controller, sensor and actuator are located at different places. Among those delays, there are two network-induced delays, one from sensor to controller and the other from controller to actuator. With the two delays considered, the closed-loop system will appear with two additive time delays in the state. Unfortunately, the two delays are generally time-varying with different properties, because the network transmission conditions is different. This implies that the systems with additive timevarying delays are more complicated and challenged. Therefore, the studies on the stability analysis and controller synthesis for MJSs with additive time-varying delay components are of theoretical and practical importance.

Fortunately, several results about Markov jump neural networks with additive time-varying delay components have been proposed in the past years. For instance, in [30], the authors investigated the synchronization of singular Markovian jumping complex dynamical networks with two additive timevarying delay components by using the pinning control. In $[31,32]$, the problem of dissipativity and passivity of Markovian jump stochastic neural networks involving two additive time-varying delays was investigated, several sufficient conditions are derived for verifying the dissipativity criteria of neural networks. [33] Concerned with the problem of finite-time boundedness, L2 gain analysis and control of Markovian jump switched neural networks with additive timevarying delays. [34] Focused on the problem of delaydependent stability and dissipativity analysis of generalized neural networks with Markovian jump parameters and two delay components. [35] Mainly concerned on stability problem of Markovian jump neural networks with mode-dependent two additive time-varying delays based on quadratic convex combination approach. Despite these efforts, however, to the best knowledge of our authors, the stabilization problem for MJSs with additive time-varying delay components has not been investigated fully in the literature.

Motivated by the aforementioned concerns, in this paper, we mainly investigate the stabilization for MJSs with additive time-varying delays. At first, we provide a novel double integral inequality, which is less conservative than the same type inequality found in the literature. Secondly, some new Lyapunov functionals with triple integral terms are constructed via considering more information about the two additive timevarying delays of system, such as $x\left(t-d_{1}(t)\right), x\left(t-d_{1}\right)$, then the 
stochastically stable condition of the Markovian jump systems in terms of linear matrix inequalities with lower conservatism is derived by using new inequalities, convex combination technique and combining with other analytical techniques. At last but not least, based on the obtained stochastically stable condition, the controller is designed later. Finally, a numerical example is given to verify the effectiveness of the proposed method and the superiority of the results.

Notation: Throughout this paper, $\mathrm{R}^{n}$ denotes the $\mathrm{n}$ dimensional Euclidean space, $\mathrm{R}^{m \times n}$ is the set of all $m \times n$ real matrices. \|\| refers to the Euclidean vector norm. For a real symmetric matrix $X, X>0(X \geq 0)$ means that $X$ is positive definite (semi-positive definite). The notation "*" in a block matrix always represents the symmetric terms. The superscript " $T$ " stands for the transpose of a matrix or a vector. $\operatorname{diag}\{\cdots\}$ for a block-diagonal matrix, and $\operatorname{sym}\{M\}=M+M^{T}$. The identity and zero matrices of appropriate dimensions are denoted by $I$ and $O$, respectively. The following nomenclature is used to simplify vector and matrix representations: $e_{i}=(0 \cdots 0, \underset{i}{I}, 0 \cdots 0),(i=1, \cdots 21)$.

\section{PROBLEM FormULATION AND PRELIMINARIES}

Consider the following MJSs with additive time-varying delays:

$$
\left\{\begin{array}{l}
\dot{x}(t)=A\left(r_{t}\right) x(t)+B\left(r_{t}\right) x(t-d(t))+C\left(r_{t}\right) u(t) \\
x\left(t_{0}+\theta\right)=\varphi(\theta), \quad \forall \theta \in[-d, 0]
\end{array}\right.
$$

where $x(t) \in R^{n}$ is the system state, $u(t) \in R^{m}$ is the control input, $\varphi(\theta)$ is an initial condition function. The time delay $d(t)=d_{1}(t)+d_{2}(t)$, where $d_{1}(t)$ and $d_{2}(t)$ represent two time-varying delays in the state with different physical characteristics and satisfy:

$$
\begin{aligned}
& 0 \leq d_{1}(t) \leq d_{1}<\infty, 0 \leq d_{2}(t) \leq d_{2}<\infty \\
& 0 \leq \dot{d}_{1}(t) \leq \mu_{1}<\infty, 0 \leq \dot{d}_{2}(t) \leq \mu_{2}<\infty
\end{aligned}
$$

where $d_{1}, d_{2}$ and $\mu_{1}, \mu_{2}$ are known constants. Naturally, we denote $d=d_{1}+d_{2}, \mu=\mu_{1}+\mu_{2} . A\left(r_{t}\right), B\left(r_{t}\right), C\left(r_{t}\right)$ are known matrix functions of the random Markov jumping process. $\left\{r_{t}, t \geq 0\right\}$ is right-continuous Markov process on the probability space taking values in a finite state space $\mathrm{N}=(1,2, \cdots, N)$ with generator $\Lambda=\left(\lambda_{i j}\right), i, j \in \mathrm{N}$ given by

$$
\operatorname{Pr}\left\{r_{t+\Delta}=j \mid r_{t}=i\right\}=\left\{\begin{array}{cc}
\lambda_{i j} \Delta+o(\Delta), & j \neq i \\
1+\lambda_{i j} \Delta+o(\Delta), & j=i
\end{array}\right.
$$

where $\Delta>0, \lim _{\Delta \rightarrow 0} o(\Delta) / \Delta=0, \lambda_{i j} \geq 0$, for $i \neq j$ is the transition rate from mode $i$ at time $t$ to mode $j$ at time $t+\Delta$, $\lambda_{i i}=-\sum_{j=1, j \neq i}^{N} \lambda_{i j}$.

For the sake of simplicity, the solution $x\left(t, \varphi(\theta), r_{0}\right)$ with $r_{0} \in \mathrm{N}$ is denoted $x(t)$. It is known from [36] that $\left\{x(t), r_{t}\right\}$ is a Markov process with an initial state $\left\{\varphi(\theta), r_{0}\right\}$ and its weak infinitesimal generator, acting on function $V\left(x(t), t, r_{t}\right)$, is defined in[37]:

$$
\begin{aligned}
L V\left(x(t), t, r_{t}\right)= & \lim _{\Delta \rightarrow 0^{+}} \frac{1}{\Delta}\left\{\varepsilon\left[V\left(x(t+\Delta), t+\Delta, r_{t+\Delta}\right) \mid x(t)\right]\right. \\
& \left.-V\left(x(t), t, r_{t}\right)\right\} .
\end{aligned}
$$

Before presenting the main results, the following definition and lemmas are necessary, which will be used in the proof of our main results.

Definition 1[38]: The system (1) with $u(t)=0$ is said to be stochastically stable if the following condition holds:

$$
\varepsilon\left\{\int_{0}^{\infty}\|x(t)\|^{2} d t \mid \varphi, r_{0}\right\} \leq \infty
$$

for every initial condition $\varphi(\theta) \in R^{n}$ and $r_{0} \in \mathrm{N}$.

Lemma 1[39]: For a positive definite matrix $R \in \mathrm{R}^{n \times n}$, and a differentiable function $\{x(u) \mid u \in[a, b]\}$, the following inequality holds:

$$
\sigma \int_{a}^{b} \dot{x}^{T}(s) R \dot{x}(s) d s \geq \Upsilon_{1}^{T} R \Upsilon_{1}+3 \Upsilon_{2}^{T} R \Upsilon_{2}+5 \Upsilon_{3}^{T} R \Upsilon_{3}
$$

where $\sigma=(b-a)$, and

$$
\begin{aligned}
& \Upsilon_{1}=x(b)-x(a), \Upsilon_{2}=x(b)+x(a)-2 \sigma^{-1} \int_{a}^{b} x(s) d s, \\
& \Upsilon_{3}=\Upsilon_{1}+6 \sigma^{-1} \int_{a}^{b} x(s) d s-12 \sigma^{-2} \int_{a}^{b} \int_{\theta}^{b} x(s) d s d \theta
\end{aligned}
$$

Lemma 2[40]: For a positive definite matrix $R \in \mathrm{R}^{n \times n}$, matrices $N_{1}, N_{2}, N_{3} \in R^{4 n \times n}$ and any differentiable function $\{x(u) \mid u \in[a, b]\}$, the following inequality holds:

$$
-\int_{a}^{b} \int_{\theta}^{b} \dot{x}^{T}(s) R \dot{x}(s) d s d \theta \leq \eta^{T} \Omega \eta,
$$

where 


$$
\begin{aligned}
\Omega= & 2^{-1} \sigma^{2} N_{1} R^{-1} N_{1}^{T}+4^{-1} \sigma^{2} N_{2} R^{-1} N_{2}^{T}+6^{-1} \sigma^{2} N_{3} R^{-1} N_{3}^{T} \\
& +\sigma \operatorname{sym}\left\{N_{1} \Pi_{1}+N_{2} \Pi_{2}+N_{3} \Pi_{3}\right\}, \quad \Pi_{1}=\bar{e}_{1}-\bar{e}_{2}, \\
\Pi_{2}= & \bar{e}_{1}+2 \bar{e}_{2}-6 \bar{e}_{3}, \quad \Pi_{3}=\bar{e}_{1}-3 \bar{e}_{2}+24 \bar{e}_{3}-60 \bar{e}_{4}, \\
\eta= & {\left[x^{T}(b), \sigma^{-1} \int_{a}^{b} x^{T}(s) d s, \sigma^{-2} \int_{a}^{b} \int_{\theta}^{b} x^{T}(s) d s d \theta,\right.} \\
& \left.\sigma^{-3} \int_{a}^{b} \int_{\theta}^{b} \int_{u}^{b} x^{T}(s) d s d u d \theta\right], \quad \bar{e}_{1}=(I, 0,0,0), \\
\bar{e}_{2}= & (0, I, 0,0), \quad \bar{e}_{3}=(0,0, I, 0), \quad \bar{e}_{4}=(0,0,0, I) .
\end{aligned}
$$

\section{MAIN RESUlts}

Our purpose in this chapter is to study the stabilization and design the controller for MJSs (1).

Theorem 1. The MJSs (1) with $u(t)=0$ is stochastically stable if there exist symmetric positive definite matrice $P_{i} \in R^{n \times n}$, $W \in R^{6 n \times 6 n}, Q_{l_{1}}, R_{l_{2}} \in R^{n \times n},\left(l_{1}=1,2,3,4,5,6 ; l_{2}=1,2,3,4,5\right)$ and any matrices $N_{l_{3}}, M_{l_{3}} \in R^{4 n \times n},\left(l_{3}=1,2,3\right), X, Y, Z, K \in R^{12 n \times n}$ such that

$$
\begin{gathered}
Q_{1}-Q_{2}>0, Q_{2}-Q_{3}>0, Q_{3}-Q_{4}>0, \\
\Psi_{20 \times 20}=\left(\begin{array}{cccc}
\Psi_{12 \times 12} & \hat{N} & \hat{M} & \hat{\Xi}_{l} \\
* & -\hat{R}_{1} & 0 & 0 \\
* & * & -\hat{R}_{2} & 0 \\
* & * & * & -\hat{R}_{31}
\end{array}\right)<0,
\end{gathered}
$$

where $(l=1,2,3,4)$ and

$$
\begin{aligned}
& \Psi_{12 \times 12}=e_{1}^{T} \Pi e_{1}+e_{1}^{T}\left(Q_{1}+Q_{5}\right) e_{1}-\left(1-\mu_{1}\right) e_{2}^{T}\left(Q_{1}-Q_{2}\right) e_{2} \\
& -(1-\mu) e_{3}^{T}\left(Q_{2}-Q_{3}\right) e_{3}-\left(1-\mu_{1}\right) e_{4}^{T}\left(Q_{3}-Q_{4}\right) e_{4} \\
& -e_{5}^{T}\left(Q_{4}+Q_{6}\right) e_{5}-e_{9}^{T}\left(Q_{5}-Q_{6}\right) e_{9}-\mathrm{H}_{11}^{T} R_{4} \mathrm{H}_{11}-3 \\
& \mathrm{H}_{12}^{T} R_{4} \mathrm{H}_{12}-5 \mathrm{H}_{13}^{T} R_{4} \mathrm{H}_{13}-\mathrm{H}_{21}^{T} R_{5} \mathrm{H}_{21}-3 \mathrm{H}_{22}^{T} R_{5} \mathrm{H}_{22} \\
& -5 \mathrm{H}_{23}^{T} R_{5} \mathrm{H}_{23}+\operatorname{sym}\left\{\zeta^{T} X\left(e_{1}-e_{2}\right)+\zeta^{T} Y\left(e_{2}-e_{3}\right)\right. \\
& \left.+\zeta^{T} Z\left(e_{3}-e_{4}\right)+\zeta^{T} K\left(e_{4}-e_{5}\right)\right\}+\operatorname{sym}\left\{e_{s i}^{T} P_{i} e_{1}\right. \\
& \left.+\dot{\eta}^{T} W \eta\right\}+d_{2} \operatorname{sym}\left\{\zeta_{10}^{T} N_{1} \zeta_{11}+\zeta_{10}^{T} N_{2} \zeta_{12}+\zeta_{10}^{T} N_{3} \zeta_{13}\right\} \\
& +d_{1} \operatorname{sym}\left\{\zeta_{20}^{T} M_{1} \zeta_{21}+\zeta_{20}^{T} M_{2} \zeta_{22}+\zeta_{20}^{T} M_{3} \zeta_{23}\right\}+e_{s i}^{T} \hat{R} \mathrm{e}_{s i}, \\
& \Pi=\sum_{j=1}^{N} \lambda_{i j} P_{j}, \hat{N}=\left(N_{1}^{T}, N_{2}^{T}, N_{3}^{T}\right), \hat{M}=\left(M_{1}^{T}, M_{2}^{T}, M_{3}^{T}\right), \\
& \hat{R}_{1}=\operatorname{diag}\left\{2 d_{2}^{-2} R_{1}, 4 d_{2}^{-2} R_{1}, 6 d_{2}^{-2} R_{1}\right\}, \hat{\Xi}_{1}=(Z, K), \hat{\Xi}_{2}=(X, Z) \text {, } \\
& \hat{R}_{2}=\operatorname{diag}\left\{2 d_{2}^{-2} R_{2}, 4 d_{2}^{-2} R_{2}, 6 d_{2}^{-2} R_{2}\right\}, \hat{\Xi}_{3}=(Y, K), \hat{\Xi}_{4}=(X, Y) \text {, } \\
& \hat{R}_{31}=\operatorname{diag}\left\{d_{2}^{-1} R_{3}, d_{1}^{-1} R_{3}\right\}, \hat{R}_{32}=\operatorname{diag}\left\{d_{1}^{-1} R_{3}, d_{2}^{-1} R_{3}\right\} \text {, } \\
& \hat{R}_{33}=\operatorname{diag}\left\{d_{2}^{-1} R_{3}, d_{1}^{-1} R_{3}\right\}, \hat{R}_{34}=\operatorname{diag}\left\{d_{1}^{-1} R_{3}, d_{2}^{-1} R_{3}\right\} \text {, } \\
& \hat{R}=\left(2^{-1} d_{2}^{2} R_{1}+2^{-1} d_{1}^{2} R_{2}+d R_{3}+d_{2}^{2} R_{4}+d_{1}^{2} R_{5}\right), e_{s i}=A_{i} e_{1}+B_{i} e_{3}, \\
& \mathrm{H}_{11}=\left(e_{9}-e_{5}\right), \mathrm{H}_{12}=\left(e_{9}+e_{5}-2 e_{6}\right), \mathrm{H}_{13}=\left(e_{9}-e_{5}+6 e_{6}-12 e_{7}\right) \text {, } \\
& \mathrm{H}_{21}=\left(e_{1}-e_{9}\right), \mathrm{H}_{22}=\left(e_{1}+e_{9}-2 e_{10}\right), \mathrm{H}_{23}=\left(e_{1}-e_{9}+6 e_{10}-12 e_{11}\right) \text {, } \\
& \zeta=\left(e_{1}, e_{2}, \cdots, e_{11}, e_{12}\right), \zeta_{10}=\left(e_{9}, e_{6}, e_{7}, e_{8}\right), \zeta_{20}=\left(e_{1}, e_{10}, e_{11}, e_{12}\right) \text {, } \\
& \zeta_{11}=\left(e_{9}-e_{6}\right), \zeta_{12}=\left(e_{9}+2 e_{6}-6 e_{7}\right), \zeta_{13}=\left(e_{9}-3 e_{6}+24 e_{7}-60 e_{8}\right) \text {, } \\
& \zeta_{21}=\left(e_{1}-e_{10}\right), \zeta_{22}=\left(e_{1}+2 e_{10}-6 e_{11}\right), \zeta_{23}=\left(e_{1}-3 e_{10}+24 e_{11}-60 e_{12}\right) \text {, } \\
& \eta=\left(e_{1}, d_{2} e_{6}, d_{2}^{2} e_{7}, d_{2}^{3} e_{8}, d_{1} e_{10}, d_{1}^{2} e_{11}, d_{1}^{3} e_{12}\right), \dot{\eta}=\left(e_{9}-e_{5}, d_{2}\left(e_{9}-e_{6}\right),\right. \\
& \left.2^{-1} d_{2}^{2}\left(e_{9}-2 e_{7}\right), e_{1}-e_{9}, d_{1}\left(e_{1}-e_{10}\right), 2^{-1} d_{1}^{2}\left(e_{1}-2 e_{11}\right)\right) \text {. }
\end{aligned}
$$

Proof. Constructing a stochastic Lyapunov functional candidate as

$$
V\left(x(t), t, r_{t}\right)=\sum_{j=1}^{5} V_{j}\left(x(t), t, r_{t}\right)
$$

where

$$
V_{1}\left(x(t), t, r_{t}\right)=x^{T}(t) P\left(r_{t}\right) x(t)+\eta^{T}(t) W \eta(t),
$$

$$
\begin{gathered}
V_{2}\left(x(t), t, r_{t}\right)=\int_{t-d_{1}(t)}^{t} x^{T}(s) Q_{1} x(s) d s+\int_{t-d(t)}^{t-d_{1}(t)} x^{T}(s) Q_{2} x(s) d s \\
\quad+\int_{t-d_{1}(t)-d_{2}}^{t-d(t)} x^{T}(s) Q_{3} x(s) d s+\int_{t-d}^{t-d_{1}(t)-d_{2}} x^{T}(s) Q_{4} x(s) d s \\
\quad+\int_{t-d_{1}}^{t} x^{T}(s) Q_{5} x(s) d s+\int_{t-d}^{t-d_{1}} x^{T}(s) Q_{6} x(s) d s \\
V_{3}\left(x(t), t, r_{t}\right)=\int_{-d}^{-d_{1}} \int_{u}^{-d_{1}} \int_{t+\theta}^{t} \dot{x}^{T}(s) R_{1} \dot{x}(s) d s d \theta d u \\
\quad+\int_{-d_{1}}^{0} \int_{u}^{0} \int_{t+\theta}^{t} \dot{x}^{T}(s) R_{2} \dot{x}(s) d s d \theta d u
\end{gathered}
$$$$
V_{4}\left(x(t), t, r_{t}\right)=\int_{-d}^{0} \int_{t+\theta}^{t} \dot{x}^{T}(s) R_{3} \dot{x}(s) d s d \theta
$$ 


$$
\begin{gathered}
V_{5}\left(x(t), t, r_{t}\right)=d_{2} \int_{-d}^{-d_{1}} \int_{t+\theta}^{t} \dot{x}^{T}(s) R_{4} \dot{x}(s) d s d \theta \\
+d_{1} \int_{-d_{1}}^{0} \int_{t+\theta}^{t} \dot{x}^{T}(s) R_{5} \dot{x}(s) d s d \theta
\end{gathered}
$$

where $P\left(r_{t}\right), W, Q_{I_{1}}, R_{l_{2}}$ have been defined in Theorem 1 and are all positive matrices. Therefore, combining the inequality (8) can we known that $V\left(x(t), t, r_{t}\right)>0$.

Then, for given $r_{t}=i \in \mathrm{N}, P_{r_{t}}=P_{i}$ and the infinitesimal operator $L$ of the stochastic process $\left\{x(t), r_{t}\right\}$ along the evolution of $V\left(x(t), t, r_{t}\right)$ are given as

$$
L V_{1}(x(t), t, i)=\xi^{T}\left[\operatorname{sym}\left\{\dot{x}^{T}(t) P_{i} x(t)+\dot{\eta}^{T} W \eta\right\}+e_{1}^{T} \Pi e_{1}\right] \xi,
$$

$$
\begin{aligned}
& L V_{2}(x(t), t, i) \leq \xi^{T}\left[e_{1}^{T}\left(Q_{1}+Q_{5}\right) e_{1}-\left(1-\mu_{1}\right) e_{2}^{T}\left(Q_{1}-Q_{2}\right) e_{2}\right. \\
& -(1-\mu) e_{3}^{T}\left(Q_{2}-Q_{3}\right) e_{3}-\left(1-\mu_{1}\right) e_{4}^{T}\left(Q_{3}-Q_{4}\right) e_{4} \\
& \left.-e_{5}^{T}\left(Q_{4}+Q_{6}\right) e_{5}-e_{9}^{T}\left(Q_{5}-Q_{6}\right) e_{9}\right] \xi,
\end{aligned}
$$

$$
\begin{aligned}
& L V_{3}(x(t), t, i)=\dot{x}^{T}(t)\left(2^{-1} d_{2}^{2} R_{1}+2^{-1} d_{1}^{2} R_{2}\right) \dot{x}(t) \\
& \quad-\int_{t-d}^{t-d_{1}} \int_{\theta}^{t-d_{1}} \dot{x}^{T}(s) R_{1} \dot{x}(s) d s d \theta-\int_{t-d_{1}}^{t} \int_{\theta}^{t} \dot{x}^{T}(s) S_{2} \dot{x}(s) d s d \theta,
\end{aligned}
$$

From Lemma 2, it follows that

$$
\begin{aligned}
& L V_{3}(x(t), t, i) \leq \dot{x}^{T}(t)\left(2^{-1} d_{2}^{2} R_{1}+2^{-1} d_{1}^{2} R_{2}\right) \dot{x}(t)+\zeta_{10}^{T}\left(2 d_{2}^{-2} N_{1}\right. \\
& \left.\quad \times R_{1}^{-1} N_{1}^{T}+4 d_{2}^{-2} N_{2} R_{1}^{-1} N_{2}^{T}+6 d_{2}^{-2} N_{3} R_{1}^{-1} N_{3}^{T}\right) \zeta_{10}+\zeta_{20}^{T}\left(2 d_{2}^{-2}\right. \\
& \left.\quad \times M_{1} R_{2}^{-1} M_{1}^{T}+4 d_{2}^{-2} M_{2} R_{2}^{-1} M_{2}^{T}+6 d_{2}^{-2} M_{3} R_{2}^{-1} M_{3}^{T}\right) \zeta_{20} \\
& \quad+d_{2} \operatorname{sym}\left\{\zeta_{10}^{T} N_{1} \zeta_{11}+\zeta_{10}^{T} N_{2} \zeta_{12}+\zeta_{10}^{T} N_{3} \zeta_{13}\right\} \\
& \quad+d_{1} \operatorname{sym}\left\{\zeta_{20}^{T} M_{1} \zeta_{21}+\zeta_{20}^{T} M_{2} \zeta_{22}+\zeta_{20}^{T} M_{3} \zeta_{23}\right\},
\end{aligned}
$$

$$
\begin{aligned}
& L V_{4}(x(t), t, i)=\dot{x}^{T}(t) d R_{3} \dot{x}(t)-\int_{t-d_{1}(t)}^{t} \dot{x}^{T}(s) R_{3} \dot{x}(s) d s \\
& \quad-\int_{t-d(t)}^{t-d_{1}(t)} \dot{x}^{T}(s) R_{3} \dot{x}(s) d s-\int_{t-d_{1}(t)-d_{2}}^{t-d(t)} \dot{x}^{T}(s) R_{3} \dot{x}(s) d s \\
& \quad-\int_{t-d}^{t-d_{1}(t)-d_{2}} \dot{x}^{T}(s) R_{3} \dot{x}(s) d s,
\end{aligned}
$$

Now, we estimate the upper bound of the last four terms on the right-hand side in inequality (19). For any matrix $X, Y, Z, K \in \mathrm{R}^{12 n \times n}$, we can get the following inequalities:

$$
\begin{aligned}
& -\int_{t-d_{1}(t)}^{t} \dot{x}^{T}(s) R_{3} \dot{x}(s) d s \leq \xi^{T}\left[d_{1}(t) \zeta^{T} X R_{3}^{-1} X^{T} \zeta+2 \zeta^{T} X\left(e_{1}-e_{2}\right)\right] \xi, \\
& -\int_{t-d(t)}^{t-d_{1}(t)} \dot{x}^{T}(s) R_{3} \dot{x}(s) d s \leq \xi^{T}\left[d_{2}(t) \zeta^{T} Y R_{3}^{-1} Y^{T} \zeta+2 \zeta^{T} Y\left(e_{2}-e_{3}\right)\right] \xi,
\end{aligned}
$$

$$
\begin{gathered}
-\int_{t-d_{1}(t)-d_{2}}^{t-d(t)} \dot{x}^{T}(s) R_{3} \dot{x}(s) d s \leq \xi^{T}\left[\left(d_{2}-d_{2}(t)\right) \zeta^{T} Z R_{3}^{-1} Z^{T} \zeta\right. \\
\left.+2 \zeta^{T} Z\left(e_{3}-e_{4}\right)\right] \xi, \quad-\int_{t-d}^{t-d_{1}(t)-d_{2}} \dot{x}^{T}(s) R_{3} \dot{x}(s) d s \\
\leq \xi^{T}\left[\left(d_{1}-d_{1}(t)\right) \zeta^{T} K R_{3}^{-1} K^{T} \zeta+2 \zeta^{T} K\left(e_{4}-e_{5}\right)\right] \xi \\
L V_{5}(x(t), t, i)=\dot{x}^{T}(t)\left(d_{2}^{2} S_{4}+d_{1}^{2} S_{5}\right) \dot{x}(t) \\
\quad-d_{2} \int_{t-d}^{t-d_{1}} \dot{x}^{T}(s) S_{4} \dot{x}(s) d s-d_{1} \int_{t-d_{1}}^{t} \dot{x}^{T}(s) S_{5} \dot{x}(s) d s,
\end{gathered}
$$

From Lemma 1, it follows that

$$
\begin{aligned}
& L V_{5}\left(x_{t}, t, i\right) \leq \dot{x}^{T}(t)\left(d_{2}^{2} R_{4}+d_{1}^{2} R_{5}\right) \dot{x}(t)-\xi^{T}\left\{\mathrm{H}_{11}^{T} R_{4} \mathrm{H}_{11}-3 \mathrm{H}_{12}^{T} R_{4}\right. \\
& \left.\mathrm{H}_{12}-5 \mathrm{H}_{13}^{T} R_{4} \mathrm{H}_{13}-\mathrm{H}_{21}^{T} R_{5} \mathrm{H}_{21}-3 \mathrm{H}_{22}^{T} R_{5} \mathrm{H}_{22}-5 \mathrm{H}_{23}^{T} R_{5} \mathrm{H}_{23} \xi\right\} .
\end{aligned}
$$

So, combining the inequality (16) to (21), it obtain that

$$
L V\left(x_{t}, t, i\right)=\sum_{j=1}^{5} L V_{j}\left(x_{t}, t, i\right) \leq \xi^{T} \Psi \xi,
$$

where

$$
\begin{gathered}
\Psi=\Psi_{12 \times 12}+\hat{N} \hat{R}_{1}^{-1} \hat{N}^{T}+\hat{M} \hat{R}_{2}^{-1} \hat{M}^{T}+d_{1}(t) X R_{3}^{-1} X^{T}+d_{2}(t) Y R_{3}^{-1} Y^{T} \\
+\left(d_{2}-d_{2}(t)\right) Z R_{3}^{-1} Z^{T}+\left(d_{1}-d_{1}(t)\right) K R_{3}^{-1} K^{T}+\Gamma^{T} \hat{R} \Gamma, \\
\xi=\left(x(t), x\left(t-d_{1}(t)\right), x\left(t-d(t), x\left(t-d_{1}(t)-d_{2}\right)\right),\right. \\
x(t-d), d_{2}^{-1} \int_{t-d}^{t-d_{1}} x(s) d s, d_{2}^{-2} \int_{t-d}^{t-d_{1}} \int_{\theta}^{t-d_{1}} x(s) d s d \theta, \\
d_{2}^{-3} \int_{t-d}^{t-d_{1}} \int_{u}^{t-d_{1}} \int_{\theta}^{t-d_{1}} x(s) d s d \theta d u, d_{1}^{-1} \int_{t-d_{1}}^{t} x(s) d s, \\
\left.d_{1}^{-2} \int_{t-d_{1}}^{t} \int_{\theta}^{t} x(s) d s d \theta, d_{1}^{-3} \int_{t-d_{1}}^{t} \int_{u}^{t} \int_{\theta}^{t} x(s) d s d \theta d u\right)^{T} .
\end{gathered}
$$

By utilizing the Schur complement and the convex combination technique given in [42], it is known that $\Psi<0$ is equivalent to the inequality (9) holds which imply that $L V\left(x_{t}, t, i\right)<0$. Therefore, using the Dynkin's formulator can we gain

$$
\varepsilon\left\{\int_{0}^{\infty}\|x(t)\|^{2} d t \mid \varphi, r_{0}\right\} \leq \infty,
$$

which means that system (1) is stochastic stable following Definition 1. The proof is completed.

Remark 1.The Lyapunov functionals we constructed in this paper consider more information about $d_{1}(t), d_{2}(t), d(t)$, such as $x\left(t-d_{1}(t)\right), x\left(t-d_{1}(t)-d_{2}(t)\right), x(t-d(t))$ and $x\left(t-d_{1}\right), x(t-d)$. In fact, the maximum of the two delays $d_{1}(t)$ and $d_{2}(t)$ may not be reached at same time, and their property may have sharply different properties, so we consider the process of the variation of delay generated in system is from $d_{1}(t)$ to $d(t)$, then to $d_{1}(t)+d_{2}$, and then to $d$, therefore the first four items in $V_{2}(x(t), t, i)$ are constructed, and the relation between the upper bound $d_{1}$ of $d_{1}(t)$ and the upper bound $d$ of $d(t)$ is also taken into account in the last two items of $V_{2}(x(t), t, i)$. Meanwhile, the integral items $\int_{t-d}^{t} \dot{x}^{T}(s) R_{3} x(s) d s$ in $L V_{4}(x(t), t, i)$ is decomposed into the form like inequality (19), it is important to 
reduce the conservation of the resulting stochastic stabile conditions.

Remark 2.It is noted that the upper bound $d$ of $d(t)$ is divided into two interval $\left[0, d_{1}\right]$ and $\left[d_{1}, d\right]$, so we construct the Lyapunov functional form like $V_{3}(x(t), t, i)$ and $V_{5}(x(t), t, i)$, and then the less conservative stochastic stable criteria is derived by utilizing the novel double integral inequality (7) and a single integral inequality (6) with less conservation. Furthermore, to increase the cross product item between the augmented vector, the last item in $V_{1}(x(t), t, i)$ is constructed, which is very useful to improve the upper bound of delay and reduce the conservation of the resulting stochastic stable criterions.

The approach of Theorem 1 can be used as a useful tool for the stabilization problem of system (1) with $u(t)=J_{i} x(t)$. Now we are in position to design the controller by convex optimization method.

Theorem 2. The system (1) with $u(t)=J_{i} x(t)$ is stochastically stable if there exist symmetric positive definite matrices $\bar{P}_{i}, \bar{Q}_{l_{1}} \in \mathrm{R}^{n \times n},\left(l_{1}=1,2,3,4,5,6\right), \bar{R}_{l_{2}} \in \mathrm{R}^{n \times n},(l=1,2,3,4,5)$, $\bar{W} \in R^{6 n \times 6 n}$, and any matrices $\bar{N}_{l_{2}}, \bar{M}_{l_{2}} \in R^{4 n \times n},\left(l_{2}=1,2,3\right)$, $\bar{X}, \bar{Y}, \bar{Z}, \bar{K} \in R^{12 n \times n}$, and a scalar $\lambda>0$, such that

$$
\begin{gathered}
\bar{Q}_{1}-\bar{Q}_{2}>0, \bar{Q}_{2}-\bar{Q}_{3}>0, \bar{Q}_{3}-\bar{Q}_{4}>0, \\
\left(\begin{array}{cc}
\bar{\Psi}_{20 \times 20} & \tilde{\Gamma}^{T} \\
* & -2 \lambda^{-1} \bar{P}+\lambda^{-2} \hat{\hat{R}}
\end{array}\right)<0,
\end{gathered}
$$

where

$$
\begin{gathered}
\bar{\Psi}_{20 \times 20}=\left(\begin{array}{cccc}
\bar{\Psi}_{12 \times 12} & \overline{\hat{N}} & \overline{\hat{M}} & \overline{\hat{\Xi}}_{l} \\
* & -\overline{\hat{R}}_{1} & 0 & 0 \\
* & * & -\overline{\hat{R}}_{2} & 0 \\
* & * & * & -\overline{\hat{R}}_{31}
\end{array}\right), \\
\bar{\Psi}_{12 \times 12}=e_{1}^{T} \bar{\Pi} e_{1}+e_{1}^{T}\left(\bar{Q}_{1}+\bar{Q}_{5}\right) e_{1}-\left(1-\mu_{1}\right) e_{2}^{T}\left(\bar{Q}_{1}-\bar{Q}_{2}\right) e_{2} \\
-(1-\mu) e_{3}^{T}\left(\bar{Q}_{2}-\bar{Q}_{3}\right) e_{3}-\left(1-\mu_{1}\right) e_{4}^{T}\left(\bar{Q}_{3}-\bar{Q}_{4}\right) e_{4} \\
-e_{5}^{T}\left(\bar{Q}_{4}+\bar{Q}_{6}\right) e_{5}-e_{9}^{T}\left(\bar{Q}_{5}-\bar{Q}_{6}\right) e_{9}-\mathrm{H}_{11}^{T} \bar{R}_{4} \mathrm{H}_{11} \\
-3 \mathrm{H}_{12}^{T} \bar{R}_{4} \mathrm{H}_{12}-5 \mathrm{H}_{13}^{T} \bar{R}_{4} \mathrm{H}_{13}-\mathrm{H}_{21}^{T} \bar{R}_{5} \mathrm{H}_{21}-3 \mathrm{H}_{22}^{T} \bar{R}_{5} \mathrm{H}_{22} \\
-5 \mathrm{H}_{23}^{T} \bar{R}_{5} \mathrm{H}_{23}+\operatorname{sym}\left\{\zeta^{T} \bar{X}\left(e_{1}-e_{2}\right)+\zeta^{T} \bar{Y}\left(e_{2}-e_{3}\right)\right. \\
\left.+\zeta^{T} \bar{Z}_{2}\left(e_{3}-e_{4}\right)+\zeta^{T} \bar{K}\left(e_{4}-e_{5}\right)\right\}+s y m\left\{e_{s i}^{T} \bar{P}_{1}+\dot{\eta}^{T} \bar{W} \eta\right\} \\
+d_{2} \operatorname{sym}\left\{\zeta_{10}^{T} \bar{N}_{1} \zeta_{11}+\zeta_{10}^{T} \bar{N}_{2} \zeta_{12}+\zeta_{10}^{T} \bar{N}_{3} \zeta_{13}\right\} \\
+d_{1} \operatorname{sym}\left\{\zeta_{20}^{T} \bar{M}_{1} \zeta_{21}+\zeta_{20}^{T} \bar{M}_{2} \zeta_{22}+\zeta_{20}^{T} \bar{M}_{3} \zeta_{23}\right\},
\end{gathered}
$$

where $(l=1,2,3,4), \zeta, \zeta_{10}, \zeta_{11}, \zeta_{12}, \zeta_{13}, \zeta_{20}, \zeta_{21}, \zeta_{22}, \zeta_{23}$ have been defined in Theorem 1. And

$$
\begin{aligned}
& \bar{\Pi}=\sum_{j=1}^{s} \lambda_{i j} \bar{P}_{j}, \overline{\hat{N}}=\left(\bar{N}_{1}^{T}, \bar{N}_{2}^{T}, \bar{N}_{3}^{T}\right), \overline{\hat{M}}=\left(\bar{M}_{1}^{T}, \bar{M}_{2}^{T}, \bar{M}_{3}^{T}\right), \\
& \overline{\hat{R}}_{1}=\operatorname{diag}\left\{2 d_{2}^{-2} \bar{R}_{1}, 4 d_{2}^{-2} \bar{R}_{1}, 6 d_{2}^{-2} \bar{R}_{1}\right\}, \overline{\hat{\Xi}}_{1}=(\bar{Z}, \bar{K}), \overline{\hat{\Xi}}_{2}=(\bar{X}, \bar{Z}), \\
& \overline{\hat{R}}_{2}=\operatorname{diag}\left\{2 d_{2}^{-2} \bar{R}_{2}, 4 d_{2}^{-2} \bar{R}_{2}, 6 d_{2}^{-2} \bar{R}_{2}\right\}, \overline{\hat{\Xi}}_{3}=(\bar{Y}, \bar{K}), \overline{\hat{\Xi}}_{4}=(\bar{X}, \bar{Y}), \\
& \overline{\hat{R}}_{31}=\operatorname{diag}\left\{d_{2}^{-1} \bar{R}_{3}, d_{1}^{-1} \bar{R}_{3}\right\}, \hat{R}_{32}=\operatorname{diag}\left\{d_{1}^{-1} \bar{R}_{3}, d_{2}^{-1} \bar{R}_{3}\right\}, \\
& \overline{\hat{R}}_{33}=\operatorname{diag}\left\{d_{2}^{-1} \bar{R}_{3}, d_{1}^{-1} \bar{R}_{3}\right\}, \hat{R}_{34}=\operatorname{diag}\left\{d_{1}^{-1} \bar{R}_{3}, d_{2}^{-1} \bar{R}_{3}\right\}, \\
& \overline{\hat{R}}=\left(2^{-1} d_{2}^{2} \bar{R}_{1}+2^{-1} d_{1}^{2} \bar{R}_{2}+d \bar{R}_{3}+d_{2}^{2} \bar{R}_{4}+d_{1}^{2} \bar{R}_{5}\right), \\
& \mathrm{H}_{11}=\left(e_{9}-e_{5}\right), \mathrm{H}_{12}=\left(e_{9}+e_{5}-2 e_{6}\right), \mathrm{H}_{13}=\left(e_{9}-e_{5}+6 e_{6}-12 e_{7}\right), \\
& \mathrm{H}_{21}=\left(e_{1}-e_{9}\right), \mathrm{H}_{22}=\left(e_{1}+e_{9}-2 e_{10}\right), \mathrm{H}_{23}=\left(e_{1}-e_{9}+6 e_{10}-12 e_{11}\right), \\
& \zeta=\left(e_{1}, e_{2}, \cdots, e_{11}, e_{12}\right), \zeta_{10}=\left(e_{9}, e_{6}, e_{7}, e_{8}\right), \zeta_{20}=\left(e_{1}, e_{10}, e_{11}, e_{12}\right), \\
& \zeta_{11}=\left(e_{9}-e_{6}\right), \zeta_{12}=\left(e_{9}+2 e_{6}-6 e_{7}\right), \zeta_{13}=\left(e_{9}-3 e_{6}+24 e_{7}-60 e_{8}\right), \\
& \zeta_{21}=\left(e_{1}-e_{10}\right), \zeta_{22}=\left(e_{1}+2 e_{10}-6 e_{11}\right), \zeta_{23}=\left(e_{1}-3 e_{10}+24 e_{11}-60 e_{12}\right), \\
& \eta=\left(e_{1}, d_{2} e_{6}, d_{2}^{2} e_{7}, d_{2}^{3} e_{8}, d_{1} e_{10}, d_{1}^{2} e_{11}, d_{1}^{3} e_{12}\right), \dot{\eta}=\left(, e_{9}-e_{5}, d_{2}\left(e_{9}-e_{6}\right),\right. \\
&\left.2^{-1} d_{2}^{2}\left(e_{9}-2 e_{7}\right), e_{1}-e_{9}, d_{1}\left(e_{1}-e_{10}\right), 2^{-1} d_{1}^{2}\left(e_{1}-2 e_{11}\right)\right) . \\
& \tilde{\Gamma}=\left(A_{1} \bar{P}_{i}+C_{i} L, 0, B_{i} \bar{P}_{i}, 0, \cdots, 0\right)_{1 \times 20}^{T},
\end{aligned}
$$

Furthermore, a desired controller gain matrix is given by

$$
J_{i}=L\left(\bar{P}_{i}\right)^{-1}
$$

Proof. When the controller $u(t)=J_{i} x(t)$, then the system (1) is formulated as follows:

$$
\dot{x}(t)=\left(A_{i}+C_{i} J_{i}\right) x(t)+B_{i} x\left(t-d_{1}(t)-d_{2}(t)\right) .
$$

Replaced $A_{i}$ with $\left(A_{i}+C_{i} J_{i}\right)$ and utilizing the Schur complement in Theorem 1, the inequality (11) can be expressed as:

$$
\Psi_{20 \times 20}+\tilde{\Gamma}^{T} \hat{R} \tilde{\Gamma}<0
$$

where $\hat{R}, \Psi_{20 \times 20}$ has been defined in Theorem 1 .

Let block diagonal matrices $L_{1}=\operatorname{diag}\left\{P_{i}^{-1}, P_{i}^{-1}, \cdots, P_{i}^{-1}\right\}, L_{2}=$ $\operatorname{diag}\left\{P_{i}^{-1}, P_{i}^{-1}, \cdots, P_{i}^{-1}\right\}$ with 20 and 6 dimensions, respectively. And $\bar{P}_{i}=P_{i}^{-1}, \bar{\Delta}=\bar{P}_{i} \Delta \bar{P}_{i}$. With these notations and (24) in mind, performing a congruence transformation to (8), (9) and $W \in R^{6 n \times 6 n}$ by $\bar{P}_{i}, L_{1}$, and $L_{2}$ respectively. Then, one can get $\bar{W}>0$, inequality (25), and the following inequality by using Schur complements,

$$
\left(\begin{array}{cc}
\bar{\Psi}_{20 \times 20} & \tilde{\Gamma} \\
* & -\hat{R}^{-1}
\end{array}\right)<0,
$$

where $\bar{\Psi}_{20 \times 20}, \tilde{\Gamma}$ and $\hat{R}$ are defined in Theorem 2. On the hand, as we all know, for any $\lambda>0$, one can note that

$$
\left(\lambda \bar{P}_{i}-\overline{\hat{R}}\right) \overline{\hat{R}}^{-1}\left(\lambda \bar{P}_{i}-\overline{\hat{R}}\right) \geq 0,
$$

it is easy to see that 


$$
-\bar{P}_{i} \overline{\hat{R}}^{-1} \bar{P}_{i} \leq-2 \lambda^{-1} \bar{P}_{i}+\lambda^{-2} \overline{\hat{R}} .
$$

Therefore, (22)-(23) hold if (25)-(27) hold. The proof is completed.

Remark 3. It is very important to introduce a positive scalar $\lambda$ to the effectiveness of Theorem 2. Otherwise, it is a high load for $-P_{i}$ to stabilize the matrices $\overline{\hat{R}}$ in (25) following the technique in [43].

\section{NUMERICAL EXAMPLES}

In order to show the reduced conservatism and the effectiveness of approaches presented in this paper, in this section, one numerical example is provided.

Example 1. To show the effectiveness of Theorem 2, consider the dynamical system (1) with $N=2$ and satisfies

$$
\begin{aligned}
& A_{1}=\left(\begin{array}{cc}
-2 & 0 \\
0 & -0.9
\end{array}\right), \quad A_{2}=\left(\begin{array}{cc}
-2 & 0 \\
0 & -9
\end{array}\right), \quad C_{1}=\left(\begin{array}{ll}
1 & 0 \\
0 & 1
\end{array}\right) \\
& B_{1}=\left(\begin{array}{cc}
-1 & 0 \\
-1 & -1
\end{array}\right), \quad B_{2}=\left(\begin{array}{cc}
-1 & 0 \\
-1 & -1
\end{array}\right), \quad C_{2}=\left(\begin{array}{ll}
1 & 0 \\
0 & 1
\end{array}\right) .
\end{aligned}
$$

If we set $\mu_{1}=0.1, \mu_{2}=0.8, d_{1}=1, d_{2}=0.988$ and $\lambda=1 / 19$. Meanwhile, the relevant transition rate matrix $\Lambda$ is considered as $\Lambda=\left(\begin{array}{cc}0.95 & 0.05 \\ 0.005 & 0.995\end{array}\right)$. According to the Theorem 2, some matrices are obtained by computing in the MATLAB as follows

$$
J_{1}=\left(\begin{array}{cc}
-1.9804 & 0.0255 \\
-3.9108 & -3.5470
\end{array}\right), \quad J_{2}=\left(\begin{array}{cc}
-1.9035 & 0.1437 \\
-1.0928 & -1.8495
\end{array}\right) .
$$

It should be pointed out that the system (1) will not be stochastic stable with the controller designed by utilizing the approach in[43], ie., when $\lambda=1$ in (27). Therefore, it is clearly that the approach in [14] for designing controller is more flexible, and this example shows the effectiveness of our methods presented in this paper.

\section{CONCLUSION}

In this paper, we study the problem of stabilization for MJSs with additive time-varying delays. Firstly, the stabilization condition of the systems is given by constructing a new Lyapunov functional with triple integral terms, using new inequalities, convex combination technique and combining with other analytical techniques. Then, the the controller of the closed-loop system is designed by the transformation technique of inequalities. Moreover, our future research topic is to obtain the less stabilization conditions of MJSs with the additive timevarying delays with fewer decision variables.

\section{ACKNOWLEDGMENT}

The authors wish to thank the editor and reviewers for a number of constructive comments and suggestions that have improved the quality of this manuscript. This work was supported by National Nature Science Foundation under Grant 11461082, 11601474, 11671206 and 11271190.

\section{REFERENCES}

[1] Krasovskii N.N. and Lidskii E.A., Analytical Design of Controllers in Systems With Random Attributes, Autom. Remote Control (Engl. Transl.), vol.22,pp.1021-1025,1961.

[2] Feng X., Loparo K. A., Ji Y. and Chizeck H. J., Stochastic stability properties of jump linear systems, IEEE Trans. Automat. Cont., vol.37, pp. 38-53,1992.

[3] Li H., Zhou Q., Chen B., Liu H., Parameter-dependent robust stability for uncertain Markovian jump systems with time delay, J. Franklin Institute, vol.38,pp.738-748,2011.

[4] Gao H., Fei Z., Lam J., Du B., Further results on exponential estimates of Markovian jump systems with mode-dependent time-varying delays, IEEETrans. Autom. Control vol.56,pp.223-229,2011.

[5] Xia J., Sun C., Zhang B., New robust $\mathrm{H} \infty$ control for uncertain stochastic Markovian jumping systems with mixed delays based on decoupling method, J. Franklin Institute, vol.349, pp.741-769,2012.

[6] Zhang B., Zheng W., Xu S., On robust $\mathrm{H} \infty$ filtering for uncertain markovian jump time-delay systems, International Journal of Adaptive Control and Signal Processing, vol.26,pp.138-157,2012.

[7] Li H., Jing X., H. Lam, Shi P., Fuzzy sampled-data control for uncertain vehicle suspension systems, IEEE Trans. Cybernetics, vol.44, pp.1111$1126,2014$.

[8] Balasubramaniam P., Revathi V.M., Ju H.P., L 2 -L $\infty$ math Con- tainer Loading Mathjax, Filtering for neutral Markovian switching system with mode-dependent time-varying delays and partially unknown transition probabilities, Applied Mathematics \& Computation, vol.219(17), pp.9524 -9542, 2013.

[9] Hale J. K., Functional Differential Equations,Springer-Verlag, New York, 1977.

[10] Xiong L.L., Cheng J., Liu X.Z., Wu T., Improved conditions for neutral delay systems with novel inequalities, The Journal of Nonlinear Sciences and Applications (JNSA), Accepted 2017.

[11] Fridman E. and Shaked U., An improved stabilization method for linear time-delay systems, IEEETrans. Autom. Control, Vol.47,pp.1931$1937,2002$.

[12] Richard J. P., Time-delay systems: An overview of some recent advances and open problems, Automat-ica, Vol.39, pp.1667-1694,2003.

[13] Wu M., He Y., She J.H. and Liu G.P., Delay-dependent criteria for robust stability oftime-varying delay systems, Automatica, Vol.40, pp.1435-1439,2004.

[14] Xiong L.L., Li Y.K., Zhou W.H., Improved Stabilization for Continuous Dynamical Systems with Two Additive Time-Varying Delays, Asian Journal of Control,Vol.17(6),pp.2229-2240,2015.

[15] Sipahi R., Vyhldal T., Niculescu S.I. and Pepe P., Time Delay Systems: Methods, Applications and New Trends, Springer-Verlag, New York, 2012.

[16] Zhang X.M., Wu M., She J.H. and He Y., Delay-dependent stabilization of linear systems with time-varying state and input delays, Automatica, Vol.41, pp.1405-1412,2005.

[17] Gao H. and Wang C., A delay-dependent approach to robust $\mathrm{H} \infty$ filtering for uncertain discrete-time state-delayed systems, IEEE Trans. Signal Process.,Vol.52,pp.1631-1640,2004.

[18] Zhu X.L. and Du X., New results of stability analysis for systems with two additive time-varying delays, Proc. 31st Chinese Control Conf., Hefei China, pp.1452-1457,2012.

[19] Xiong L.L., Zhong S.M. and Tian J.K., New robust stability condition for uncertain neutral systems with discrete and distributed delays, Chaos Solitons Fractals, Vol.42,pp.1073-1079,2009.

[20] Xiong L.L., Zhong S.M. and Li D.Y., Novel delay-dependent asymptotical stability of neutral systems with nonlinear perturbations, J. Comput.Appl. Math., Vol. 232, pp. 505-513,2009.

[21] Tian J.K., Xiong L.L., Liu J.X. and Xie X.J., Novel delay-dependent robust stability criteria for uncertain neutral systems with time-varying delay, Chaos Solitons Fractals, Vol.40,pp. 1858-1866,2009. 
[22] Xiong L.L., Tian J.K., Liu X.Z., Stability analysis for neutral Markovian jump systems with partially unknown transition probabilities, J. Frankl. Inst.-Eng. App. Math., Vol.349,pp.2193-2214,2012.

[23] Yu H.G., Zhong S.M., Agarwal R. P. and Xiong L.L., Species permanence and dynamical behavior analysis of an impulsively controlled ecological system with distributed time delay, Comput. Math.Applicat., Vol.59, pp.3824-3835,2010.

[24] Yang J., Zhong S.M. and Xiong L.L., A descriptor system approach to non-fragile control for uncertain fuzzy neutral systems, Fuzzy Sets Syst., Vol.160,pp.423-438,2009.

[25] Long S.H., Zhong S.M., Zhu H. and Xiong L.L., Delay-dependent stochastic admissibility for a class of discrete-time nonlinear singular Markovian jump systems with time-varying delay, Commun. Nonlinear Sci. Numer. Simul., Vol.19,pp.673-685,2014.

[26] Cao J.D. and Wang J., Delay-dependent robust stability of uncertain nonlinear systems with time delay, Appl. Math. Comput., Vol.154, pp.289-297,2004.

[27] Lam J., Gao H. and Wang C., Stability analysis for continuous system with two additive time-varying delay components, Syst. Control Lett., Vol.56, pp.16-24,2007.

[28] Tian J.K. and Zhong S.M., Improved delay-dependent stability criteria for neural networks with two additive time-varying delay components, Neurocomputing, Vol.77,pp.114-119,2012.

[29] Xiao N. and Ji Y.M., New approaches on stability criteria for neural networks with two additive time-varying delay components, Neuro computing, Vol.118,pp.150-156,2013.

[30] Rakkiyappan R., Kaviarasan B., Rihan F. A., Lakshmanan S., Synchronization of singular Markovian jumping complex networks with additive time-varying delays via pinning control. Journal of the Franklin Institute, Vol. 352(8), pp.3178-3195,2015.

[31] Nagamani G., Radhika T., Zhu Q., An Improved Result on Dissipativity and Passivity Analysis of Markovian Jump Stochastic Neural Networks With Two Delay Components. IEEE Transactions on Neural Networks \& Learning Systems, Vol.99, pp.1-14,2016.

[32] Nagamani G, Radhika T., Dissipativity and Passivity Analysis of Markovian Jump Neural Networks with Two Additive Time-Varying Delays. Neural Processing Letters, pp.1-22,2015.

[33] Ali M.S., Saravanan S., Cao J., Finite-time boundedness, L2-gain analysis and control of Markovian jump switched neural networks with additive time-varying delays. Nonlinear Analysis Hybrid Systems, Vol.23,pp.27-43,2017.

[34] Chen G., Xia J., Zhuang G., Delay-dependent stability and dissipativity analysis of generalized neural networks with Markovian jump parameters and two delay components. Journal of the Franklin Institute, Vol. 353(9), pp.2137-2158,2016.

[35] Muthukumar P., Subramanian K., Stability criteria for Markovian jump neural networks with mode-dependent additive time-varying delays via quadratic convex combination. Neurocomputing, Vol.205, pp.75-83, 2016.

[36] Xu S.Y., Mao X.R., Delay-dependent $\mathrm{H} \infty$ control and filitering for uncertain Markovian jump system with time-varying delay, IEEE Transactions on Circuits and Systems Part I: Regular Papers, Vol.54(9), pp.2070-2077,2007.

[37] Zhang Y., He Y., Wu M., She J.H., Stability and $\mathrm{H} \infty$ performance analysis for markovian jump systems with time-varying delay and partial information on transition probabilities. IFAC Proceedings Volumes, Vol. 44(1), pp.8669-8674,2011.

[38] Skorohod A.V., Asymptotic Methods in the Theory of Stochastic Differential Equation, American Mathematical Society, Providence, RI 1989.

[39] Park P.G., Lee W.I., Lee S.Y., Auxiliary function-based integral inequalities for quadratic functions and their applications to time-delay systems. Journal of the Franklin Institute, Vol.352(4), pp.1378$1396,2015$.

[40] Zhao N., Lin C., Chen B., Wang Q., A new double integral inequality and application to stability test for time-delay systems, Applied Mathematics Letters, Vol.65, pp.26-31,2017.
[41] H. Zeng, Y. He, M. Wu, J. She. New results on stability analysis for systems with discrete distributed delay, Automatica, Vol.60,pp.189192,2015 .

[42] Zhu X.L., Yue D., Wang Y., Delay-dependent stability analysis for neural networks with additive time-varying delay components. Control Theory \& Applications Iet, Vol. 7(3), pp.354-362,2013.

[43] Shao H.Y. and Zhang Z.Q., Stability and stabilization for systems with two additive time-varying delay components, Proc. 30 th Chinese Control Conf.,Yantai, China, pp.1119-1124,2011. 